\title{
A experiência de estudantes de Odontologia com dilemas éticos
}

Lorena Marques da Nóbrega*; Ítalo de Macedo Bernardino**; Kevan Guilherme Nóbrega Barbosa***; Pierre Andrade Pereira de Oliveira ${ }^{* * * *}$; Rilva Suely de Castro Cardoso Lucas ${ }^{* * * *}$; Sérgio d'Ávila ${ }^{* * * *}$

\footnotetext{
* Doutorando em Odontologia, Universidade Estadual da Paraíba - UEPB

** Graduando em Odontologia, Universidade Estadual da Paraíba - UEPB

*** Doutorando em Odontologia, Universidade Federal de Minas Gerais - UFMG

Professores doutores, Disciplina de Saúde Coletiva, Universidade Estadual da Paraíba - UEPB.
}

\section{RESUMO}

A universidade é um espaço privilegiado para formação de pessoas e neste processo são evidenciadas questões sociais, humanas e éticas. Na Odontologia estas se estabelecem individualmente entre profissionais e pacientes e, coletivamente, entre a prática profissional e a sociedade. Este estudo do tipo transversal avaliou a experiência de estudantes de Odontologia de uma universidade pública com os dilemas éticos vividos durante sua formação acadêmica. Para tanto foram entrevistados 64 alunos do $4^{\circ}$ e $5^{\circ}$ ano do curso. Observou-se uma ligeira predominância de alunos do sexo masculino $(50,7 \%)$, com idade entre 19 e 22 anos. Entre os pesquisados, a maioria afirmou ter tido contato com a teoria da ética profissional durante a graduação. Um quarto dos alunos entrevistados confirmou ter passado por alguma situação eticamente conflitante; todos consideraram importante a discussão de conteúdos que reflitam a bioética no currículo; e grande parte dos alunos que afirmaram ter vivenciado algum conflito ético eram do sexo masculino e cursavam o $5^{\circ}$ ano. Foi verificado que os dilemas éticos são percebidos pelos alunos e vivenciados ainda durante sua formação acadêmica.
Descritores: Ética Odontológica. Bioética. Estudantes de Odontologia.

\section{INTRODUÇÃO}

Entre os desafios educacionais da Odontologia é relevante destacar a importância do ensino da ética e deontologia durante o processo de formação profissional $^{1,2}$. Para tanto, a ética no exercício da profissão se desenvolve na formação profissional direcionada para as atitudes, valores e habilidades que são construídos no exercício desta prática ${ }^{3}$.

As clínicas de ensino odontológico apresentam no seu dia a dia algumas situações características, que tendem a potencializar os problemas e conflitos que normalmente fazem parte da rotina das instituições que prestam atendimento em saúde à população. $\mathrm{O}$ atendimento a pacientes por parte dos estudantes não infringe os princípios éticos de relacionamento, desde que se tenha certeza de que os pacientes estão devidamente informados sobre a realidade das instituições, que estes não sejam submetidos a riscos desneces- 
sários e que existam regras rigorosas quanto aos princípios do respeito à autonomia do paciente e da confidencialidade das informações obtidas durante o tratamento ${ }^{4}$.

A relação entre o profissional de saúde e o seu paciente se faz por um processo de interação social, por meio de um sistema que define o papel dos participantes, especifica o padrão de comportamento e fornece um conjunto de valores e orientações, em termos dos quais, os membros interatuantes são motivados para ação. $\mathrm{O}$ êxito dessa situação traduz-se na eficiência da atenção em saúde e está diretamente relacionado com as atividades, valores, conhecimentos e expectativas dos participantes. Esses valores e padrões sobre a saúde pertencentes à cultura é que são os determinantes da ação que leva ao início do contato profissional-paciente. $\mathrm{O}$ paciente, ele mesmo, tem dificuldade em decidir se está ou não doente, e neste último caso qual a atitude a ser tomada ${ }^{5}$.

O dia a dia do exercício da Odontologia é caracterizado por dilemas éticos ocasionados por questões contemporâneas: o atendimento a pacientes soropositivos para o vírus da imunodeficiência humana (HIV), o comércio de dentes humanos, as pesquisas com novos procedimentos e biomateriais, as contradições entre políticas públicas de saúde e justiça social, a constante busca da humanização e a importância dos princípios éticos na relação entre o profissional e o seu paciente. Assim, o futuro profissional a ser formado dentro dessa realidade deve possuir visão integral do paciente, agregando à sua formação a competência ética ${ }^{6,7}$.

É importante observar e identificar, a partir da visão dos futuros profissionais, os problemas e conflitos éticos vivenciados nas clínicas dos cursos de Odontologia, buscando conhecer suas causas e a maneira como eles enfrentam estes dilemas. Este estudo visa avaliar as situações de dilemas éticos vivenciados por estudantes de Odontologia de uma universidade pública.

\section{MATERIAIS E MÉTODOS}

O estudo foi realizado em Campina Grande, um município de porte médio do Nordeste Brasileiro e que apresenta população de 385.276 mil habitantes, considerada como um dos principais polos de desenvolvimento econômico do interior do Nordeste. Faz parte de uma região Metropolitana formada por 23 municípios cuja população estimada é de 687.545 habitantes $^{8}$.

O curso de Odontologia da Universidade Estadual da Paraíba (UEPB) é integralizado no período de dez semestres. Os estudantes entram em contato com pacientes no $4^{\circ}$ semestre, destacando-se que $80 \%$ da carga horária dos dois últimos anos consiste em atendimentos clínicos.

O presente estudo quantitativo, com desenho do tipo transversal, compreendeu acadêmicos que cursavam os quatro últimos períodos do curso. Os semestres foram agrupados em anos: quarto e quinto ano. Esse grupo foi escolhido por ser o que já possuía experiência com a prática na clínica odontológica. Segundo dados da coordenação do curso, haviam 296 alunos devidamente matriculados no curso de Odontologia, sendo que 104 estavam cursando os dois últimos anos, sendo 50 no $5^{\mathrm{a}}$ ano e 54 no $4^{\mathrm{a}}$ ano.

A pesquisa foi cadastrada na Plataforma Brasil, avaliada e aprovada pelo Comitê de Ética em Pesquisa e recebeu o CAAE $n^{\circ}$ 5712.5.0000.5187. Foram observados os aspectos éticos com base nas legislações nacionais (Resolução 466/12) e internacionais (Declaração de Helsinque, versão 2002). 
A amostra foi probabilística do tipo casual simples ou aleatória simples. Foram excluídos aqueles alunos que por três vezes consecutivas não foram encontrados e os recusaram a firmar o Termo de Consentimento Livre e Esclarecido.

Um estudo piloto $(n=16)$ com alunos concluintes foi realizado em dezembro de 2013 com a finalidade de validar o instrumento de coleta e verificar o tempo necessário para a aplicação do questionário. Estes sujeitos não foram incluídos no estudo.

A coleta de dados foi realizada por meio de um questionário elaborado pelos pesquisadores, constituído de dados demográficos (idade e sexo), ano do curso e participação em estágio extramuros, informações em relação às situações de dilemas éticos vivenciados pelos pesquisados e foi aplicado por apenas um entrevistador, em junho de 2014.

Os dados foram armazenados em planilha e o software estatístico utilizado foi o Statistical Package for the Social Sciences (SPSS) versão 20.0. O nível de significância utilizado nas decisões dos testes estatísticos foi de 5,0\%. Para a análise dos dados, foram obtidas distribuições absolutas e percentuais uni e bivariadas das variáveis na escala nominal das medidas estatísticas: valor mínimo, valor máximo, média, desvio padrão e coeficiente de variação para as variáveis numéricas (técnicas de estatística descritiva). Foram utilizados os testes estatísticos Quiquadrado de Pearson e Teste Exato de Fisher.

\section{RESULTADOS}

A taxa de resposta ao estudo foi de $72,1 \%(n=75)$, composta por 42 alunos do $5^{\circ}$ ano e 33 do $4^{\circ}$ ano. A idade dos participantes variou de 19 a 30 anos, com média de 24 anos.

Na tabela 1 apresenta-se a distribui- ção por sexo, faixa etária, ano do curso e participação em estágio extramuros. Pouco mais que a maioria da amostra foi composta por alunos do sexo masculino $(50,7 \%)$, a faixa etária mais prevalente foi a de 22 a 24 anos, representando $44,0 \%$ dos pesquisados e mais da metade dos alunos não participava de estágios fora do ambiente da universidade $(50,7 \%)$, sendo $56,0 \%$ dos indivíduos do $5^{\circ}$ ano.

A tabela 2 apresenta a distribuição das opiniões quanto à importância do conteúdo da Bioética e de situações conflitantes. Quando perguntados se eles já haviam tido contato com o conteúdo do tema, 88,0\% afirmaram que sim. Para 20,0\% da amostra, já houve alguma situação eticamente conflitante. Destes, $60,0 \%$ relataram ter vivenciado conflito gerado pela divergência de conteúdo entre os professores. Quando perguntados se o curso incentivava a discussão de novos conteúdos da ética, 76,0\% afirmaram haver esse tipo de discussão durante os anos de graduação. Todos os entrevistados consideraram importante a inclusão da temática no curriculum do curso de graduação.

A tabela 3 relaciona o gênero e o ano de curso dos estudantes pesquisados e sua vivência de algum dilema ético. Foi encontrada diferença estatisticamente significativa ( $p<0,05$ - teste exato de Fisher). Quando se associou o ano do curso com a vivência de algum dilema ético, ficou evidenciado que a maioria dos estudantes que relataram ter vivenciado alguma situação conflitante cursa o $5^{\circ}$ ano.

\section{DISCUSSÃO}

O conhecimento dos fundamentos teóricos e práticos da ética é de importância para a formação do profissional em saúde, pois 
Tabela 1 - Dados sócio demográficos dos entrevistados

\begin{tabular}{lcc}
\hline Variável & n & \% \\
\hline Sexo & 38 & 50,7 \\
Masculino & 37 & 49,3 \\
Feminino & 75 & 100,0 \\
Total & & \\
Idade & 22 & 29,3 \\
19 a 21 & 33 & 44,0 \\
22 a 24 & 20 & 26,7 \\
25 a 30 & 75 & 100,0 \\
Total & & \\
Alunos & 33 & 44,0 \\
$4^{\circ}$ ano & 42 & 56,0 \\
$5^{\circ}$ ano & 75 & 100,0 \\
Total & & \\
Estágio extramuros & 37 & 49,3 \\
Sim & 38 & 50,7 \\
Não & 38 & 100,0 \\
Total & 75 & \\
\hline
\end{tabular}

Tabela 2 - Distribuição dos entrevistados quanto a conteúdos de bioética, situações conflitantes, discussão de conteúdos e importância da bioética

\begin{tabular}{lcc}
\hline Variável & n & \% \\
\hline Teve aulas com o conteúdo de bioética? & 66 & 88,0 \\
Sim & 09 & 12,0 \\
Não & 75 & 100,0 \\
Total & & \\
Já passou por alguma situação eticamente conflitante? & 15 & 20,0 \\
Sim & 60 & 80,0 \\
Não & 75 & 100,0 \\
Total & & \\
Qual a situação conflitante? & 09 & 60,0 \\
Conflito de conteúdo entre os professores & 04 & 26,7 \\
Desconhecimento da rotina pelos alunos & 02 & 13,3 \\
Recusa em atender pacientes HIV & 15 & 100,0 \\
Total & & \\
O curso incentiva a discussão de novos conteúdos da bioética? & 57 & 76,0 \\
Sim & 18 & 24,0 \\
Não & 75 & 100,0 \\
Total & & \\
Considera importante a bioética no currículo? & 75 & 100,0 \\
Sim & 0 & 0,0 \\
Não & 75 & 100,0 \\
\hline Total & & \\
\hline
\end{tabular}


Tabela 3 - Relação entre dilema ético, gênero e ano de curso

\begin{tabular}{|c|c|c|c|c|c|}
\hline \multirow[b]{3}{*}{ Variável } & \multicolumn{4}{|c|}{ Dilema Ético } & \multirow[b]{3}{*}{$\mathbf{p}$} \\
\hline & \multicolumn{2}{|c|}{ Sim } & \multicolumn{2}{|c|}{ Não } & \\
\hline & $\mathbf{n}$ & $\%$ & $\mathbf{n}$ & $\%$ & \\
\hline Sexo & & & & & 0,249 \\
\hline Masculino & 10 & 66,7 & 28 & 46,7 & \\
\hline Feminino & 05 & 33,3 & 32 & 53,3 & \\
\hline Total & 15 & 100,0 & 60 & 100,0 & \\
\hline Ano & & & & & $0,045^{*}$ \\
\hline $4^{\circ}$ ano & 03 & 20,0 & 30 & 50,0 & \\
\hline $5^{\circ}$ ano & 12 & 80,0 & 30 & 50,0 & \\
\hline Total & 15 & 100,0 & 60 & 100,0 & \\
\hline
\end{tabular}

favorece a compreensão da realidade e encaminha as discussões sobre os dilemas éticos da profissão ainda durante a graduação. $\mathrm{O}$ ambiente dos cursos superiores é ideal para essas discussões em virtude de ser o local que estão sendo formados os futuros profissionais responsáveis pelo cuidado e a atenção em saúde.

$\mathrm{O}$ ensino da deontologia junto com a formação universitária é considerado um compromisso da profissão para promover o comportamento ético dos futuros profissionais. Avanços em ambos os conteúdo indicam que, em muitas escolas de Odontologia, essa formação está sendo ensinada cedo e muitas vezes em um formato que enfatiza auto-reflexão e raciocínio moral ${ }^{9}$.

Em relação ao gênero, os resultados obtidos apontam uma ligeira predominância de homens; estes achados são diferentes de outros estudos ${ }^{10,11}$ e da tendência nacional. Nos Estados Unidos o número de mulheres tem crescido na escolha da profissão ${ }^{12}$.

Nesse estudo os pesquisados apresentavam idade entre 20 e 30 anos, a mesma faixa etária relatada por Oliveira et al., (2002) $)^{13}$, sendo a média de idade em torno dos 24 anos, um pouco acima dos resultados encontrado em outro estudo no Brasil ${ }^{10}$.

O aprendizado não se dá somente por meio dos processos de diagnóstico e cura das doenças, mas também pela vivência dos problemas. Pouco mais que a metade dos estudantes que participaram da pesquisa $(50,7 \%)$, afirmou ter participado de algum tipo de estágio extracurricular, porém quando perguntados sobre onde ocorreram os dilemas, todos afirmaram ser no âmbito da universidade, a mesma situação encontrada por Susan et al. (2009) ${ }^{14}$.

No curso pesquisado existe uma única disciplina com o conteúdo de deontologia, que enfoca os compromissos éticos e morais dos futuros profissionais e que é ministrada no quinto semestre do curso, diferente de outras escolas, cujos alunos discutem os problemas éticos já no primeiro ano do curso $^{2}$. Seria interessante que o conteúdo fosse diluído no decorrer do curso e que mais disciplinas pudessem discutir os compromissos éticos durante o exercício profissional. Odom et al. $(2000)^{15}$ estudaram os aspectos éticos em 4 escolas de Odontologia dos Estados Unidos e observaram que a ênfase educacional sobre a ética aparece no decorrer do primeiro ano do currículo.

A graduação de profissionais experientes e qualificados em Odontologia é uma condição necessária, mas não suficiente para garantir a qualidade de cuidados de saúde 
bucal. O outro requisito é o compromisso para aplicar as habilidades com a integridade moral, prestação de cuidados adequados e de qualidade no melhor interesse dos pacientes. A boa prática da Odontologia depende de indivíduos comprometidos a tratar seus pacientes e a sociedade de forma justa ${ }^{16}$.

Assim, o profissional formado dentro dessa realidade deve ter uma visão integral do paciente, incorporando à sua formação a competência ética. A construção da formação moral e ética deficitária pode trazer consequências para a sociedade quando o estudante passa desta condição à de profissional formado, prestando atendimento odontológico à população com base em valores invertidos, priorizando leis e regras em detrimento da vida e dos direitos humanos ${ }^{6}$.

Com referência à questão do contato com a bioética durante a formação profissional, $88 \%$ dos estudantes afirmou que tiveram algum contato com a bioética no decorrer do curso universitário, porém a grande maioria afirma que o curso não oferece os subsídios para a discussão de novos conteúdos da bioética, nem são realizadas discussões acerca dos problemas existentes, corroborando com o estudo realizado por Sharp et al. $(2005)^{2}$.

Dentro de instituições de assistência médica que ensinam futuros profissionais por meio do atendimento a pacientes surgem, frequentemente, conflitos morais e dilemas éticos. É importante refletir sobre as diferentes questões éticas envolvidas na relação terapêutica protagonizada pelo paciente, o aluno e o professor. Dentre estes questionamentos, destacam-se: Qual a autonomia dessas pessoas durante o atendimento? Podem recusar determinados procedimentos? Como se processa, na relação paciente/aluno/ professor, o confronto de diferentes inte- resses: de um lado, a necessidade terapêutica; de outro, o interesse acadêmico? A formação de futuros profissionais é a importante missão das instituições de ensino ${ }^{4}$.

Neste estudo pôde-se perceber que a parcela maior dos estudantes que mencionaram ter passado por alguma situação que configurasse dilema ético, pertencia ao $5^{\circ}$ ano, não sendo uma surpresa, pois tal resultado pode ser atribuído ao fato de tais estudantes estarem cursando a última série, apresentando maior vivência clínica e, consequentemente, maior chance de terem presenciado tal situação, além de estarem mais próximos do final do curso e do enfrentamento da realidade de se ver sozinho dentro de um consultório, resolvendo os problemas que venham a ocorrer.

Verificou-se nesse grupo de estudantes que $20 \%$ haviam passado por alguma situação conflitante, diferente dos achados do estudo de Susan et al., (2009) ${ }^{14}$, cujo relato de conflito ético esteve presente em quase $100 \%$ da amostra.

As clínicas odontológicas de ensino têm no seu cotidiano algumas situações peculiares que tendem a potencializar problemas e conflitos que normalmente fazem parte da rotina de instituições que prestam atendimento de saúde à população através de estudantes. Isto acontece principalmente porque as pessoas ali atendidas são colocadas na condição, mesmo temporária, de objeto de ensino para os futuros profissionais ${ }^{4}$.

Esse tema vem assumindo significância ainda maior nas últimas décadas, já que o ensino odontológico se depara com um importante desafio: prover aos acadêmicos do curso uma formação, pautada em preceitos éticos e humanistas em tempos de intenso desenvolvimento, novas abordagens terapêuticas como a biotecnologia e a terapia genética 
e da necessidade de superar dilemas éticos no atendimento a pacientes com doenças infecciosas, como as pessoas HIV positivas $^{17,18}$.

O motivo de conflito mais citado pelos estudantes foi aquele gerado pela forma diferente de ministrar o mesmo conteúdo entre os professores. A Odontologia conta com variedades de técnicas que possibilitam resultados finais semelhantes, propondo um mesmo objetivo.

Essa variação ocorre em virtude da escola seguida e estudada pelo professor, experiência clínica e senso crítico do mesmo. $\mathrm{O}$ uso de protocolos clínicos para procedimentos, elaborados em comum acordo pelos professores das disciplinas, sendo adaptados a individualidade de cada caso conforme necessário no devido momento do atendimento, facilitaria o melhor esclarecimento para o aluno acerca dos procedimentos e técnicas a serem utilizados, principalmente nessa fase inicial, até que o aluno venha a desenvolver seu senso crítico e passe a ter maior experiência e maturidade para vislumbrar essas variações.

A percepção de estudantes sobre as questões éticas decorrentes dos dilemas que ocorrem na prática odontológica é de grande relevância para a experiência de um estudante de um curso que visa promover a saúde e o bem-estar da população.

Muito se discute no meio acadêmico sobre de que maneira e em que medida deve se dar a educação ética nos cursos de graduação em Odontologia, porém há consenso sobre a sua relevância e a necessidade de não apenas estar presente na retórica sobre o perfil desejável do egresso, mas também de modo objetivo na estrutura curricular. Esses dados irão contribuir para a discussão de como resolver os dilemas éticos que vão acompanhar a vida dos profissionais de Odontologia.

Os futuros profissionais buscam por uma especialização precoce ainda durante a graduação. Isto demostra uma visão fragmentada do curso, mesmo com a emergente possibilidade de inserção de recém-graduados no serviço público.

Assim sendo, é preciso resgatar a função social que a profissão requer e a necessidade de formação com a ênfase generalista $^{19}$.

Embora tenha sido reformulado recentemente, o Código de Ética Odontológica em vez de focar o paciente como sujeito do processo de cuidados de saúde, continua se concentrando no profissional e é predominantemente voltado para questões legalistas e corporativistas, não discutindo de forma abrangente os aspectos relacionados aos dilemas éticos ${ }^{20,21}$.

Desta forma, existe a necessidade de interpor, longitudinalmente, do início ao final do curso, os conteúdos de Bioética perpassando outras matérias, uma vez que se trata da necessidade de formar profissionais cada vez mais éticos e comprometidos com a assistência e o cuidado humanizado da população.

\section{CONCLUSÃO}

De acordo com o propósito do estudo e considerando as condições de coleta de dados da pesquisa, é possível concluir que o aluno tem contato com a teoria da ética profissional durante a graduação, que percebe e já vivenciou dilemas éticos durante a formação, sendo o conflito mais relatado aquele relacionado com as diferentes formas de ministrar o mesmo conteúdo entre os professores. 


\section{ABSTRACT \\ The experience of dental students with ethical dilemmas}

The university is a privileged space to train people with a look at the reality. In this process, the social, human and ethical issues are evident. In Dentistry, ethical issues are individually established among professionals and patients, and collectively between the professional practice and society. This crosssectional study evaluated the experience of Dental at a public university with ethical dilemmas experienced during their academic training. We interviewed 64 students who attended the 4 th and 5 th year of the course. It was observed a slight frequency of males (50.7\%), predominantly aged between 19 and 22 years. Among those surveyed, most reported having had contact with the theory of professional ethics during graduation. One quarter of the students interviewed confirmed having gone through a situation ethically conflicting, all considered important the discussion of contents reflecting the bioethics in the curriculum, much of students who reported having experienced any ethical conflict were male and were attending the 5th grade. It was found that ethical dilemmas are perceived and experienced by students even during their academic formation.

Descriptors: Dental, Ethics. Bioethics. Dental, Students.

\section{REFERÊNCIAS}

1. Schwartz B. An innovative approach to teaching ethics and professionalism. J Can Dent Assoc. 2009;75(5):338-40.

2. Sharp HM, Kuthy RA, Heller KE. Ethical dilemmas reported by fourth-year dental students. J Dent Educ. 2005;69(10):111622.

3. David WC. The role of empathy in Ethics. J Dent Educ. 2010;74(12):1275-81.

4. Gonçalves ER, Verdi MIM. Ethical problems in patient care at a dental school clinic. Cien e Saude Colet. 2007;12(3):55-64.

5. Meira AR. Bioética e vulnerabilidade: o médico e o paciente. Rev Assoc Med Bras. 2004;50(3):249-51.

6. Freitas SFT, Kovaleski DF, Boing AF. Moral development of graduates from a dentistry course: a constructivist evaluation. Cien Saude Colet. 2005; 10(2):453-62.

7. Leão HMC. A importância das teorias éticas na prática da bioética. Rev Bras Saúde Mater Infant. 2010;10(suppl.2) :427-32.

8. Instituto Brasileiro de Geografia e Estatística (IBGE). Primeiros resultados do Censo 2010. Disponível em: http://www.censo2010.ibge.gov.br/dados divulgados/Index.php?uf=25

9. Berk NW. Teaching Ethics in Dental Schools: trends, techniques, and targets. J Dent Educ. 2001;65(8):744-50.

10. Blue CM. Do Dental Hygiene Students fit the learning profile of the Millennial Student? J Dent Educ. 2009;73(12) :1372-8

11. Mariño RJ, Morgan MV, Winning T, Thomson WM, Marshall RI, Gotjamanos $\mathrm{T}$, et al. Socio demographic backgrounds and career decisions of Australian and New Zealand dental students. J Dent Educ. 2006;70(2):169-78.

12. González G, Anderson E, Novak KF. Valachovic RW. U.S. Dental School Applicants and Enrollees 2009 entering class. J Dent Educ. 2011;75(8):113357.

13. Oliveira FT de, Sales-Peres A, SalesPeres SH de C, Yarid SD, Silva RHA. Ética odontológica: conhecimento de acadêmicos e cirurgiões-dentistas sobre os aspectos éticos da profissão. Rev Odontol UNESP. 2008;37(1):33-9

14. Susan ID, Ed D, Peter G, Fitzpatrick R, Ed D, Zornosa X, et al. Dental Hygiene Students' attitudes toward ethical dilemmas in practice. J Dent Educ. 2009;7(3):345-57.

15. Odom JG, Beemsterboer PL, Pate TD, Haden NK. Revisiting the status of dental ethics instruction. $J$ Dent Educ. 
2000;64(12):772-4.

16. Silva RHA, Sales-Peres A, Almeida CT, Sales-Peres SHC. O estudante de Odontologia e a questão dos estágios. Rev ABENO. 2007;7(1):13-9.

17. Acharya S. The Ethical climate in academic dentistry in India: faculty and student perceptions. J Dent Educ. 2005; 69(6):671-80.

18. Senna MIB, Guimarães MDC, Pordeus IA. Factors associated with dentists' willingness to treat HIV/AIDS patients in the National Health System in Belo Horizonte, Minas Gerais, Brazil. Cad Saude Publ. 2005;21(1):217-225.

19. Barbosa KGN, Dias JN, Cavalcante GMS, Nóbrega LM, Granville-Garcia AF, d'Avila S. Formação e perspectiva do mercado de trabalho sob o olhar de alunos de Odontologia. Pesqui Bras Odontopediatria Clin Integr. 2013; 13(1):89-94.
20. Pyrrho M, Prado MM, Cordón J, Garrafa V. Bioethical analysis of the Brazilian Dentistry Code of Ethics. Cien e Saude Colet. 2009;14(5):1911-8.

21. Brasil, Conselho Federal de Odontologia (CFO). Código de Ética Odontológica. [acesso em 04 jan 2016]. Disponível em: http://cfo.org.br/wp-content/uploads/ 2009/09/codigo_etica.pdf.

Correspondência para:

Sérgio d'Avila

davila2407@hotmail.com

Av. das Baraúnas, 351, Bairro Universitário. 58429-500 Campina Grande - PB 